\title{
LEGION-Based Automatic Road Extraction From Satellite Imagery
}

\author{
Jiangye Yuan, DeLiang Wang, Fellow, IEEE, Bo Wu, Lin Yan, and Rongxing Li
}

\begin{abstract}
An automatic method for road extraction from satellite imagery is presented. The core of the proposed method is locally excitatory globally inhibitory oscillator networks (LEGION). The road extraction task is decomposed into three stages. The first stage is image segmentation by LEGION. In the second stage, the medial axis of each segment is computed, and the medial axis points corresponding to narrow regions are selected. The third is the road grouping stage. Alignment-dependent connections between selected points are established, and LEGION is utilized to group well-aligned points, which represent the extracted roads. Due to the selective gating mechanism of LEGION, different roads in an image are grouped separately. Road extraction results on synthetic and real images are presented. A comparison with other methods shows that the proposed method produces very competitive extraction results.
\end{abstract}

Index Terms-Locally excitatory globally inhibitory oscillator networks (LEGION), medial axis transform, oscillatory correlation, road extraction.

\section{INTRODUCTION}

$\mathbf{R}$ OAD extraction from digital imagery has been intensively studied in the computer vision and remote sensing fields. It is largely motivated by the rapidly increasing volume of collected imagery, which makes manual processes exceedingly time consuming. Many techniques have been proposed, and they are classified according to whether an approach requires human intervention. In semiautomatic approaches, a human operator provides information such as starting points or starting directions, which provide critical assistance in tracking roads [2], [16], [28]. By contrast, automatic approaches attempt to achieve true operational autonomy. Although reliable performance is more difficult to achieve, automatic approaches still attract a great deal of research due to potential productivity gains [1], [4]-[6], [19].

Manuscript received September 21, 2009; revised November 8, 2010 and February 1, 2011; accepted March 31, 2011. Date of publication May 31, 2011; date of current version October 28, 2011. This work was supported in part by the National Geospatial-Intelligence Agency University Research Initiatives under Grant HM 1582-07-1-2027.

J. Yuan and D. Wang are with the Department of Computer Science and Engineering and the Center for Cognitive Science, The Ohio State University, Columbus, OH 43210 USA (e-mail: yuanj@cse.ohio-state.edu; dwang@cse.ohio-state.edu).

B. Wu was with the Mapping and GIS Laboratory, The Ohio State University, Columbus, OH 43210 USA. He is now with the Department of Land Surveying and Geo-Informatics, The Hong Kong Polytechnic University, Kowloon, Hong Kong (e-mail: 1sbowu@polyu.edu.hk).

L. Yan, and R. Li are with the Mapping and GIS Laboratory, Department of Civil and Environmental Engineering and Geodetic Science, The Ohio State University, Columbus, OH 43210 USA (e-mail: yan.351@ osu.edu; li.282@osu.edu).

Digital Object Identifier 10.1109/TGRS.2011.2146785
Objects appear in natural scenes as groups of similar sensory features. Gestalt psychology reveals a set of principles to guide the grouping process based on local features. Elements tend to be perceptually grouped if they are close to each other (proximity), are similar to one another (similarity), form a smooth and continuous curve (good continuation), or have similar temporal behaviors (common fate) [20]. Since roads in satellite imagery tend to have uniform features that are distinct from neighboring regions, it is reasonable to expect that they can be automatically extracted using Gestalt grouping principles.

Dynamical systems represent a promising approach to object segmentation [14]. In particular, locally excitatory globally inhibitory oscillator networks (LEGION) proposed by Terman and Wang provide a framework to group object features and segment different objects through oscillatory correlation [21], [23]. The oscillatory correlation theory asserts that oscillators corresponding to the pixels of the same object synchronize, and those corresponding to pixels of different objects desynchronize. It has been shown that a LEGION network consisting of relaxation oscillators can rapidly achieve synchronization within a locally coupled oscillator assembly and desynchronization between different assemblies. LEGION has been successfully applied to a number of scene analysis tasks, including image segmentation, object selection, and speech segregation [23].

In this paper, a new automatic approach to road extraction using LEGION dynamics is presented. This approach is based on the observation that roads are relatively thin and homogeneous regions with smooth directional changes and large gray level differences with surrounding regions. The method has three stages: 1) image segmentation using a LEGION network; 2) medial axis extraction within each segment and selection of points located in potential road areas; and 3) road medial axis point grouping using a LEGION model with alignmentdependent connections.

The remainder of this paper is organized as follows. In Section II, the architecture and dynamics of LEGION are reviewed. In Section III, the method comprising segmentation, selection, and road grouping is presented. In Section IV, experimental results and comparisons are reported. Conclusions and further discussion are in Section V. This paper is an extended version of a conference paper [27].

\section{LEGION MODEL}

LEGION is a network of Terman-Wang oscillators [14]. An oscillator $i$ is defined as a feedback loop between an excitatory 


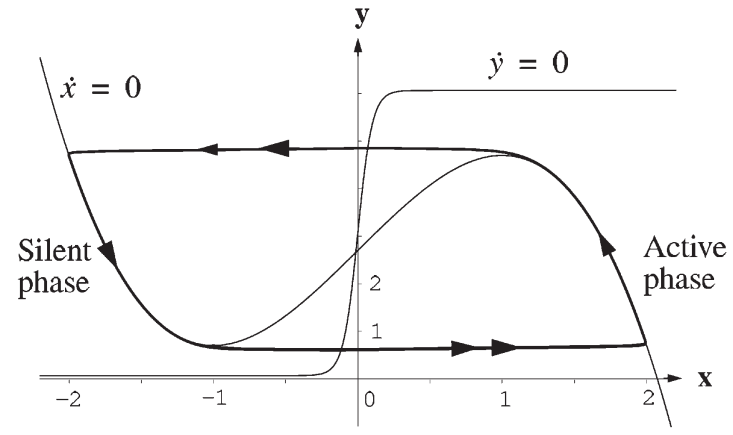

(a)

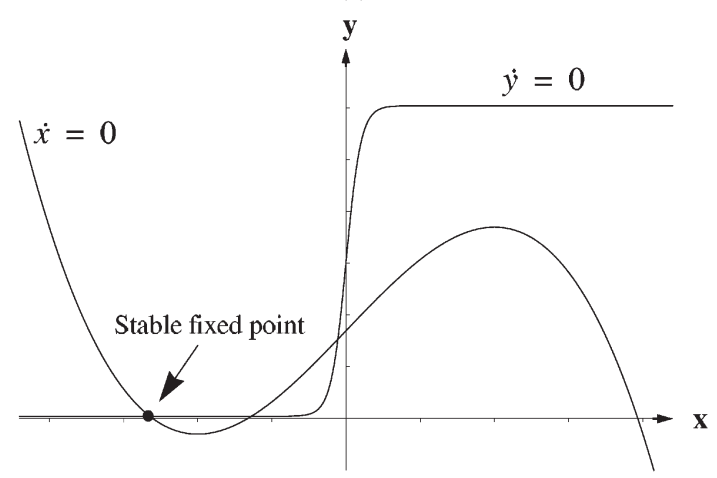

(b)

Fig. 1. Dynamics of a single oscillator. (a) Behavior of an enabled oscillator, which produces a limit cycle trajectory. The arrows indicate the motion direction. (b) Behavior of an excitable oscillator. In this case, it approaches a stable fixed point.

unit $x_{i}$ and an inhibitory unit $y_{i}$

$$
\begin{aligned}
& \dot{x}_{i}=3 x_{i}-x_{i}^{3}+2-y_{i}+I_{i}+S_{i}+\rho \\
& \dot{y}_{i}=\varepsilon\left(\alpha\left(1+\tanh \left(x_{i} / \beta\right)\right)-y_{i}\right)
\end{aligned}
$$

where $I_{i}$ and $S_{i}$ denote external stimulation and overall internal input from other parts of the network, respectively, and $\rho$ is a noise term. The variable $\varepsilon$ is set to be a very small positive number. In this case, (1) defines a typical relaxation oscillator, as illustrated in Fig. 1. The $x$-nullcline of the oscillator is a cubic, and the $y$-nullcline is a sigmoid. When $I_{i}>0$, these nullclines intersect along the middle branch of the cubic and the oscillator is enabled [see Fig. 1(a)]. When $I_{i}<0$, the oscillator approaches a fixed point [seeFig. 1(b)]; in this situation, the oscillator is in an excitable state. An excitable oscillator could be activated if it receives large enough overall input $S_{i}$. When the oscillator becomes oscillatory, its periodic orbit alternates between a silent phase, corresponding to low $x$ activity, and an active phase, corresponding to high $x$ activity. The dynamic evolution of the oscillator proceeds slowly within the silent or the active phase, while the alternation between the two phases occurs on a fast time scale, referred to as jumping. $\alpha$ is a parameter to control the relative durations of the two phases, and $\beta$ determines the steepness of the sigmoid.

The simplest LEGION network is a 2-D grid, as shown in Fig. 2. An oscillator is connected to its four nearest neighbors, where the connections are all excitatory. A global inhibitor receives excitation from every oscillator in the network and

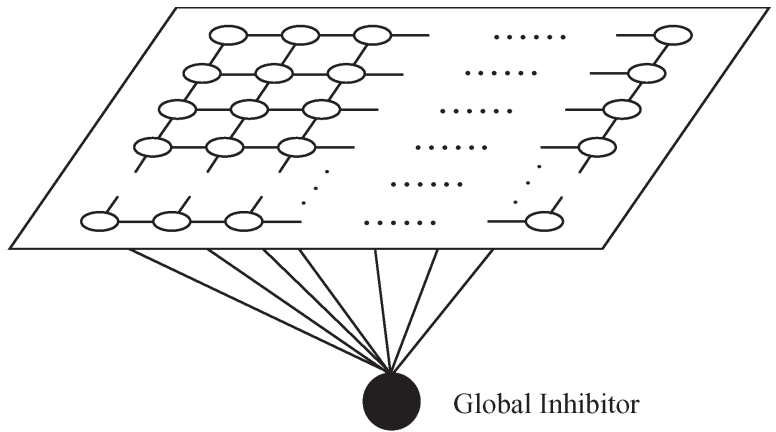

Fig. 2. Architecture of a 2-D LEGION network.

inhibits all the oscillators. The coupling term $S_{i}$ in (1a) is then defined as

$$
S_{i}=\sum_{j \in N(i)} W_{i j} H\left(x_{j}-\theta_{x}\right)-W_{z} H\left(z-\theta_{z}\right) .
$$

Here, $W_{i j}$ is a connection weight that determines the amount of excitation that oscillator $j$ sends to oscillator $i$, and $N(i)$ is the set of the neighboring oscillators that connect to $i$, the size of which can be chosen differently for specific tasks. $H$ denotes the Heaviside step function. Both $\theta_{x}$ and $\theta_{z}$ are thresholds. An oscillator sends excitation to its neighbors, and the global inhibitor exerts inhibition, when their activities exceed their respective thresholds. $W_{z}$ is the weight of inhibition from the global inhibitor $z$, whose activity is defined as

$$
\dot{z}=\phi\left(\sigma_{\infty}-z\right)
$$

where $\phi$ is a parameter and $\sigma_{\infty}$ equals one if $x_{i} \geq \theta_{x}$ for at least one oscillator and zero if otherwise.

The basic dynamics of LEGION can be briefly described as follows. Once an oscillator is in the active phase, it triggers the global inhibitor, which attempts to inhibit the entire network. The inhibition prevents the oscillators of different assemblies from jumping up but does not affect the oscillators of the same assembly because of local excitation. An enabled oscillator propagates its excitation to its neighbors and, from them, to its further neighbors until all the oscillators of the same assembly are active. Thus, the dynamics of LEGION is a process of both synchronization and desynchronization, achieved respectively by local cooperation through excitatory coupling and global competition via the global inhibitor [21].

\section{LEGION-BASED ROAD EXTRACTION}

In this section, the proposed approach for road extraction is presented, which is summarized in Fig. 3. After LEGIONbased image segmentation, the medial axis points of segments are extracted, and candidate road points are selected. By establishing alignment-dependent connections between candidate points, LEGION is further used to group the points representing roads. While LEGION has been utilized for image segmentation previously [9], [12], it has not been explored for road extraction. 


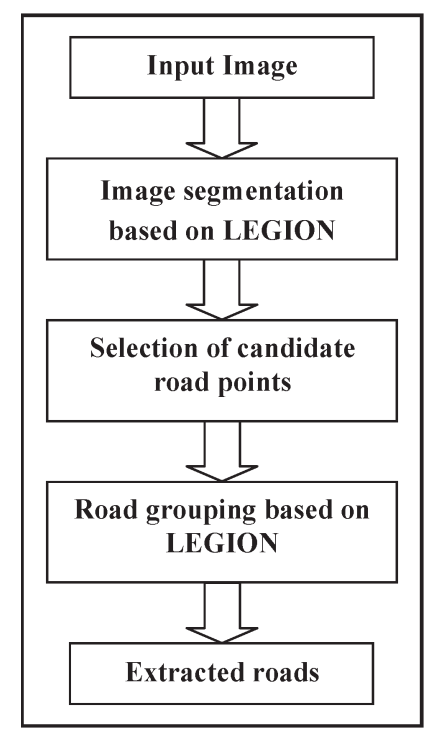

Fig. 3. Flow diagram of the proposed system for automatic road extraction.

\section{A. Segmentation}

For processing a 2-D image, each oscillator in a LEGION network corresponds to a pixel of the image and is connected to its eight nearest neighbors. The connection weight between two adjacent oscillators is set to $W_{i j}=I_{M} /\left(1+\left|I_{i}-I_{j}\right|\right)$, where $I_{i}$ is the value of pixel $i$ and $I_{M}$ is the maximum pixel value [24].

When handling real images, image noise can cause fragmentation, which impairs LEGION segmentation. To address this problem, a lateral potential for each oscillator is introduced [24]. When an oscillator receives large excitation from its neighborhood, it has a high lateral potential. Such an oscillator is called a leader. A major oscillator block must contain at least one leader, while a noisy fragment does not contain a leader. In the segmentation process, leaders are stimulated to activate oscillator groups. All the fragments correspond to oscillators that cannot sustain oscillations and are hence considered as background. Moreover, to facilitate computation, an algorithm following the main steps of LEGION dynamics has been abstracted. A detailed description of this algorithm can be found [24].

In order to provide better segmentation for road extraction, a new step is introduced to produce more roadlike segments with well-localized boundaries. Roads in a digital image appear as thin and elongated regions. Since leaders are required to be at the center of large homogeneous regions, roads rarely contain leaders and thus tend to be segmented to the background. For example, Fig. 4(b) shows a segmentation result of the image in Fig. 4(a). In Fig. 4(b), each segment is labeled by a distinct gray level. As can been seen, while the major regions are segmented, roads are put into the background, indicated by black areas. To obtain road segments, each background pixel is treated as an oscillator and fed into a LEGION network with a different coupling term where an oscillator is activated if its corresponding pixel value is close to the mean gray value of the active oscillators of a neighboring assembly [8]. Each background oscillator is stimulated, and if a stimulated

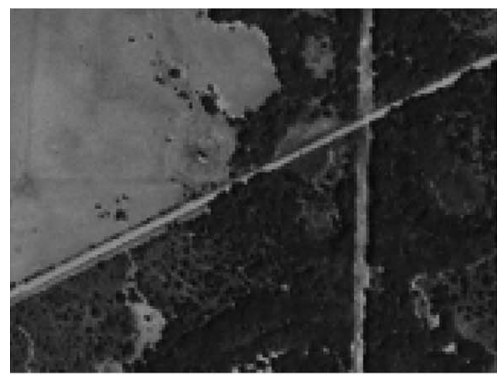

(a)

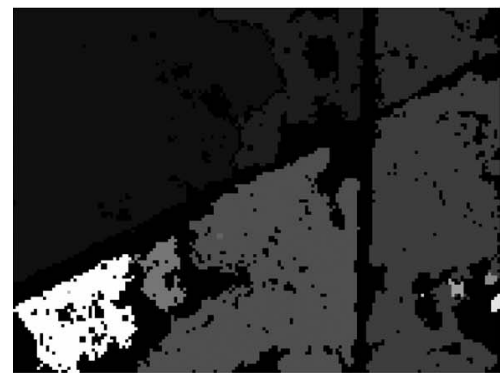

(b)

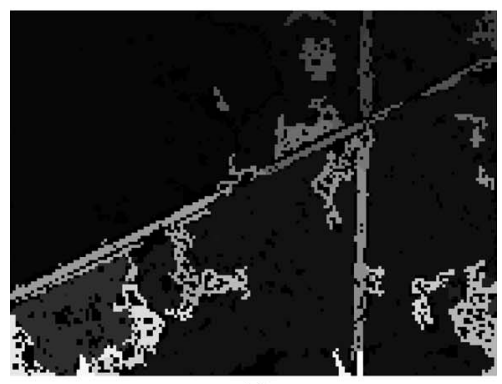

(c)

Fig. 4. LEGION image segmentation. (a) Satellite image containing roads. (b) Result of LEGION segmentation where each gray level indicates a distinct segment. (c) Result after the step of obtaining road segments.

oscillator can recruit a large enough oscillator assembly, the corresponding area is considered as a new segment.

Roads typically have clear boundaries. In order to obtain accurate road segments, boundary information is integrated in the step introduced earlier. By applying a Laplacian of the Gaussian filter to an image, the pixels on both sides of the boundaries have large filter responses with opposite signs, while the pixels within the boundaries have small responses. When a stimulated oscillator propagates its excitation to its neighboring oscillators, the filter responses of the corresponding pixels are checked, and thresholding is applied so that the propagation stops at the pixels immediately outside the boundaries. In particular, for roads brighter than the surroundings, the propagation stops if filter responses are greater than a small positive number, whereas for roads darker than the surroundings, the propagation stops if filter responses are smaller than a small negative number. Whether roads are brighter or darker than the surroundings depends on the surface materials. For example, asphalt roads appear dark and cement roads appear bright. The result after this step with bright road thresholding is shown in Fig. 4(c), where road segments are attained. Note that, at this stage, a road will correspond to multiple disconnected segments for many real images. 


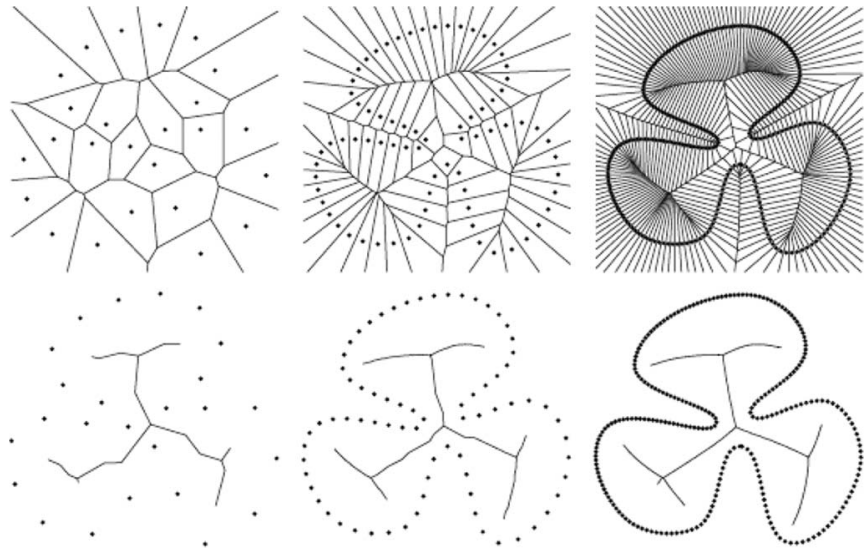

Fig. 5. Medial axis approximated by the Voronoi diagram (from [3]). In each column, the top is the Voronoi diagram for a set of samples on the boundary, and the bottom is the Voronoi vertices and edges lying inside the shape.

In summary, segmentation is performed in two steps. The first step is designed for regions and produces large and smooth segments. The second step is designed to be sensitive to boundaries which tend to be missed in the first step, and this step segments narrow areas with clear edges.

\section{B. Selection}

After segmentation of an image, the segments that correspond to roads should be selected. The medial axis transform is employed to achieve this goal [6]. The concept of the medial axis transform is to transform a 2-D object into a 1-D line representation that largely preserves the extent and connectivity of the original object. The medial axis of an object is defined as the loci of the centers of all circles that touch the boundaries at two or more points. The radius of the circle recorded at a point of the medial axis provides information about local thickness. The shape of the original object can be recovered by plotting circles at all the medial axis points.

A common approach to compute the medial axis is the Voronoi diagram [3], [10], [17]. Given a set of points $S$ in a plane, the Voronoi diagram is a partition of the plane into cells, each of which contains all the points in the plane closer to one particular point in $S$ than to any others. It has been pointed out that the vertices of the Voronoi diagram for a set of samples on a boundary curve in the object approximate the medial axis [3]. The more densely the space is sampled, the more accurate the medial axis is, as illustrated in Fig. 5.

A closing operation is first performed to smooth the boundary - closing is defined as a dilation operation where each background pixel next to an object pixel is changed into an object pixel, followed by an erosion operation where each object pixel next to a background pixel is changed into a background pixel. By treating the boundary pixels of segments as the samples, the medial axis points of each segment are obtained from the Voronoi diagram. Each medial axis point with its radius indicates the position and size of the region that it lies in. Since road regions are characterized by their narrow widths, a medial axis point is selected as a candidate if its radius is sufficiently small. The radius threshold is determined

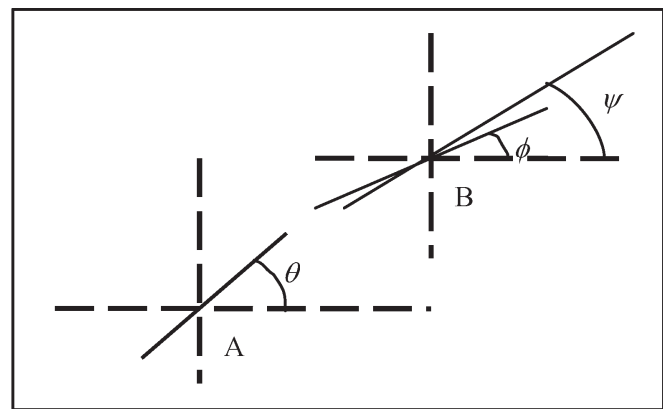

(a)

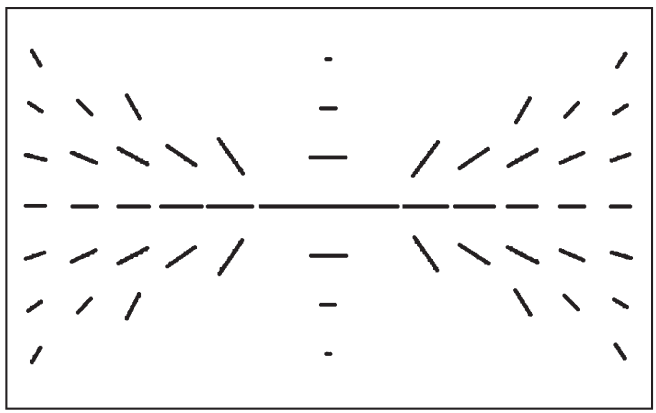

(b)

Fig. 6. Illustration of horizontal connections. (a) Cocircularity constraint. $\theta$ and $\phi$ are the tangents to the circle passing through $A$ and $B$. (b) Connectivity pattern of a horizontally oriented cell (from [26]). The cell is located at the center of the image. The orientations of the lines represent the preferred orientations, and the length of a line indicates connection strength.

by image resolution and target road width. Medial axis points are selected instead of segments because there are segments that partially belong to roads due to segmentation error, and thus, selection based on medial axis points gives more accurate results.

\section{Road Grouping}

After selection, the medial axis points corresponding to roads are mostly extracted. However, there are nonroad points that are also selected due to their small radii. In order to group road points and eliminate nonroad points, a LEGION model with alignment-dependent connections is proposed.

In an image containing oriented elements, some sets of elements tend to be perceptually grouped [26]. It has been suggested that this perceptual effect is mediated by long-range horizontal connections within the visual cortex, which are dependent on the positions of and orientations between the elements [13], [26]. For an oriented element, there exist two types of excitatory connections, one spreading along the axis of the element orientation, called coaxial, and another limited to a narrow zone orthogonal to the orientation axis, called transaxial [26].

The coaxial connection is a pattern similar to the cocircular connectivity pattern [18], as shown in Fig. 6(a). For the element at location $A$ with local orientation $\theta$, there is a preferred orientation $\phi$ for the element at location $B$, which is determined by the following rule. Given orientation $\theta$ and locations $A$ and $B$, a unique circle exists which passes through $A$ and $B$ and has a tangent orientation at $A$ identical to $\theta$. Orientation $\phi$ is 
specified by the tangent to the circle at $B$, computed by the following equation [26]:

$$
\phi=2 \tan ^{-1}\left(\frac{y}{x}\right)-\theta
$$

where $(x, y)$ are the coordinates of $B$ with the origin at $A$. If the local orientation at $B$, denoted by $\psi$, accords with the preferred orientation $\phi, A$ receives the strongest excitation from $B$. As $\psi$ deviates from $\phi$, the connection weight falls off following a Gaussian function. The connection weight also decreases as a Gaussian function of the distance between $A$ and $B$. Note that the coaxial connections are confined to low curvature deviations from the orientation axis.

For transaxial connection, the preferred orientation of an element is parallel to that of the target element. This set of connections is more spatially sensitive. The weights decrease as the angle deviation increases much more quickly than those of coaxial connections.

The connectivity pattern of a horizontally oriented element is illustrated in Fig. 6(b), which shows the preferred orientations and connection strengths. The connection weight between two elements can be written as suggested in [26]

$$
W=G\left(|\psi-\phi|_{\text {acute }}, \sigma_{\psi}\right) \times G\left(\sqrt{x^{2}+y^{2}}, \sigma_{d}\right)
$$

where $|\psi-\phi|_{\text {acute }}$ denotes the acute angle between $\psi$ and $\phi, G$ stands for a Gaussian function, and $\sigma_{\psi}$ and $\sigma_{d}$ are set differently for coaxial and transaxial connections.

Under the assumption that road segments are aligned collinearly or curvilinearly (i.e., on a smooth curve), a new LEGION model with long-range horizontal connections is proposed and applied to selected medial axis points in order to simulate the perceptual "pop-out" for well-aligned points and thus implement road segment grouping.

Medial axis points, which are available from the previous stage, are considered as oscillators. Due to the 1-D nature of a medial axis, the orientation of each oscillator can be readily calculated from its neighborhood. The coupling term $S_{i}$ of oscillator $i$ takes the following form:

$$
S_{i}=\operatorname{Max}_{j \in N(i)}\left\{W_{i j} H\left(x_{j}-\theta_{x}\right)\right\}-W_{z} H\left(z-\theta_{z}\right)
$$

where $W_{i j}$ is determined by the long-range horizontal connection, and $N(i)$ is a window centered at $i$ with a window size of $\left(2 \sigma_{d}+1\right) \times\left(2 \sigma_{d}+1\right)$. Here, instead of summation, a maximization operation is adopted, which focuses on the pairwise relations of oscillators [24]. The maximization operation ensures that each oscillator, whether the corresponding medial axis point is in the middle or the end of a road, has the same overall effective connection weight from its neighbors.

Similar to applying LEGION to image segmentation, leaders need to be selected and stimulated to avoid fragmentation. Here, the lateral potential of an oscillator is defined as the total connection weights that it receives through long-range horizontal connection within its neighborhood. If an oscillator has a large enough lateral potential from its neighbors, then it becomes a leader. The neighborhood size and potential threshold are two tunable parameters, where the neighborhood size can be differ- ent from $\left(2 \sigma_{d}+1\right) \times\left(2 \sigma_{d}+1\right)$. Large neighborhood size and potential threshold result in leaders that lie in longer and more smoothly elongated roads.

By LEGION dynamics, oscillators corresponding to the medial axis points of one road are synchronized, and those corresponding to the medial axis points of different roads are desynchronized. The oscillators that are not well aligned with any of the leaders remain excitable throughout the process. As a result, the medial axis points representing roads are extracted. The extracted points have a dense distribution, resulting in road centerlines. Based on the extracted points, the road regions can be easily recovered. A pixel is labeled as a road region if it is in the same segment as a medial axis point and within the circle defined by the radius recorded at that point.

The selection stage produces some medial axis points corresponding to roadlike objects, e.g., sidewalks and trees, along road sides. Since they are close and parallel to roads, they are likely to be grouped into the resulting roads. In order to exclude those nonroad points, the cue that roads have distinct gray value is incorporated into the grouping stage. When a leader in road regions is stimulated, it will only recruit the oscillator with a similar pixel value, where the threshold is the same as in the step of obtaining road segments. Hence, sidewalks and trees with different pixel values can be excluded in this manner.

\section{EXPERIMENTAL RESULTS AND COMPARISONS}

\section{A. Synthetic Image Example}

To illustrate the behavior of the LEGION network with longrange horizontal connections, a $50 \times 50$ oscillator network has been simulated. A synthetic input image is shown in Fig. 7(a). There are three patterns, which consist of discontinuous but smoothly aligned points. These points can be considered as the medial axis points of different road segments.

In the simulation, all oscillators corresponding to the object pixels are stimulated with $I=2.0$. The differential equations are solved using the fourth-order Runge-Kutta method with the following parameter settings: $\varepsilon=0.02, \alpha=5.0, \beta=0.1$, $\theta_{x}=-0.5, \theta_{z}=0.1, \phi=10.0$, and $W_{z}=3.0$. The step size is 0.2 . The orientation of each oscillator is approximated by the line that passes through the oscillator and its nearest neighbor. For computing connection weights, $\sigma_{\psi}=20^{\circ}$ and $\sigma_{d}=30$. The instantaneous activity of the network at different evolution stages is shown in Fig. 7(b)-(e). The gray value of each pixel is proportional to $\left(x-x_{\min }\right) /\left(x_{\max }-x_{\min }\right)$, where $x_{\max }$ and $x_{\min }$ are the maximal and minimal $x$ values of all the oscillators. It can be seen that the oscillators representing each pattern reach their active phase and are separate from the rest of the image. This successive "pop-out" of the patterns continues in a stable periodic way. The temporal evolution of all the oscillators is shown in Fig. 7(f). The activities of the oscillators of each pattern are combined as one trace. The effect of synchrony and desynchrony can be clearly observed.

\section{B. Satellite Images}

Next, the results of applying the method to real satellite images are shown. To alleviate the computational burden when 


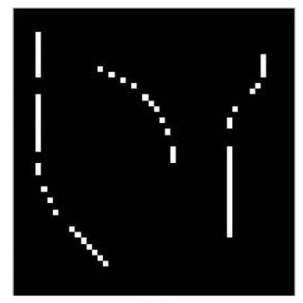

(a)

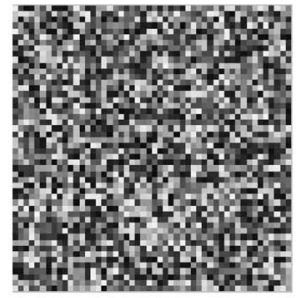

(b)

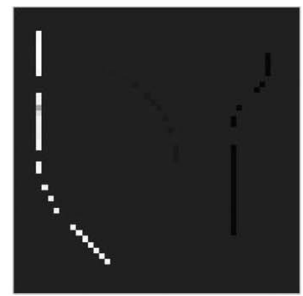

(c)

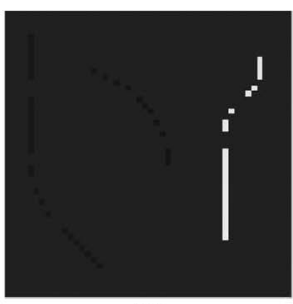

(d)

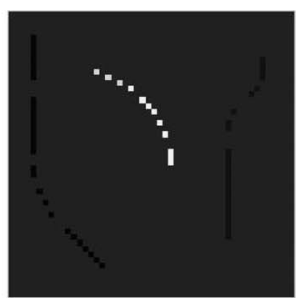

(e)

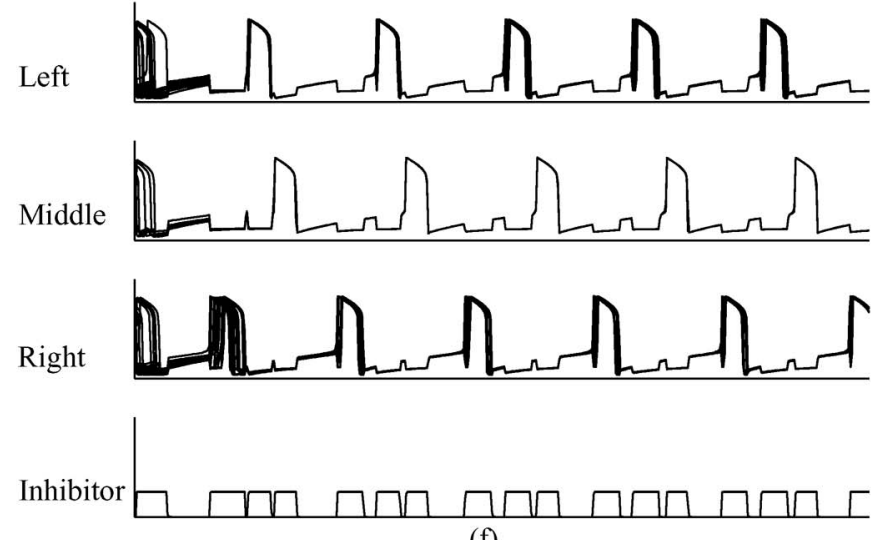

(f)

Fig. 7. Road segment extraction. (a) $50 \times 50$ synthetic image containing three interrupted roads. (b) Snapshot at the beginning of dynamic evolution showing random phases of the corresponding oscillators. (c-e) Sequence of snapshots after (b) showing extracted road segments. (f) Upper three traces show the combined $x$ activities of all the oscillators, where the oscillators representing the same pattern are combined in one trace. The bottom trace shows the activity of the global inhibitor.

processing large real images, the abstracted algorithm of LEGION mentioned earlier [24] is adopted in the first and third stages. To accurately detect the local orientation of an oscillator, the angles of the lines passing through the oscillator and its neighbors are calculated in an $11 \times 11$ window. The angles are rounded to the nearest $10^{\circ}$, and the orientation of the oscillator is set to the angle shared by the most neighbors. If the neighbors sharing the angle are fewer than one-tenth of all the neighbors, the oscillator is eliminated.

As explained earlier, two kinds of filter response thresholding are applied for dark and bright roads, respectively. Since an automatic method cannot involve human intervention, both kinds of thresholding are applied to an image to produce two segmentation results. Through bright road thresholding, few and relatively small dark road segments are obtained, which can rarely generate leaders of dark roads in the road grouping stage. As a result, bright road thresholding tends to only produce bright roads. Even if some leaders of dark roads are selected, the extracted dark roads usually lie within those produced via dark road thresholding. The same analysis applies for dark road thresholding. Hence, the final extraction is simply given by combining the two sets of results.

A number of parameters are used in the system. In LEGION segmentation, a leader generation method proposed in [9] is adopted, in which three parameters are involved. $R_{p}$ is the size of the neighborhood. $T_{\mu}$ and $T_{\sigma}$ are two thresholds to reflect region homogeneity. For the experiments in this paper, they are fixed as $R_{p}=3, T_{\mu}=0.1$, and $T_{\sigma}=2.0 . W_{z}$ is the inhibition weight, the choice of which has been discussed at length [24]. For the step of obtaining road segments, the threshold of the gray value difference is set to 20 , and the minimal size of a new segment is set to 20 pixels.

Six parameters are involved in computing long-range horizontal connections. For both coaxial and transaxial connections, $\sigma_{\psi}$ denotes the standard deviation of the angle Gaussian function, $\sigma_{d}$ denotes the standard deviation of the distance Gaussian function, and $K$ denotes the maximum curvature deviation allowed in order to establish the connections. These parameters capture the curvature tolerance of extracted roads. In the experiments, fixed values are used: For coaxial connections, $\sigma_{\psi}=20^{\circ}, \sigma_{d}=30$, and $K=15^{\circ}$, and for the transaxial ones, $\sigma_{\psi}=10^{\circ}, \sigma_{d}=5$, and $K=10^{\circ}$.

There are different types of roads. For example, interstate roads are more important to extract than field paths. Important roads appear longer and change directions more smoothly. Ideally, a system should be able to generate multiple sets of roads according to their importance. This flexibility can be reflected to a certain degree by the parameters of neighborhood size $N_{p}$ and potential threshold $\theta_{p}$ used in leader selection. Tight parameter constraints lead to fewer leaders with more prominent roads, which are generally of high importance. On the other hand, a loose threshold results in more leaders with smaller roads and also introduces more false alarms that are roadlike regions.

$W_{z g}$ is the inhibition weight in the LEGION network for road grouping. Too large a value may cause incomplete extracted roads, while too small a value may include nonroad pixels. Although, in the experiments, this parameter is slightly adjusted to produce more accurate results, a fixed value of 0.85 works well. 


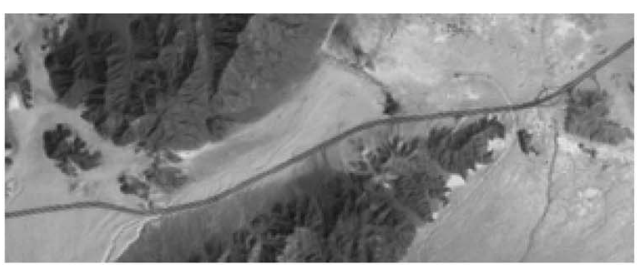

(a)

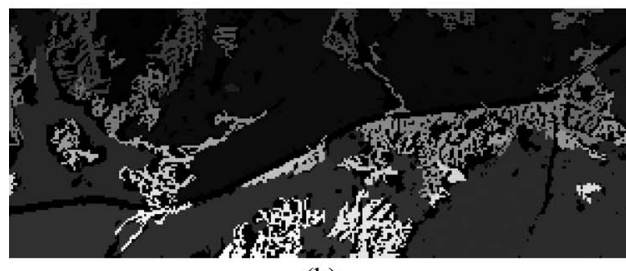

(b)

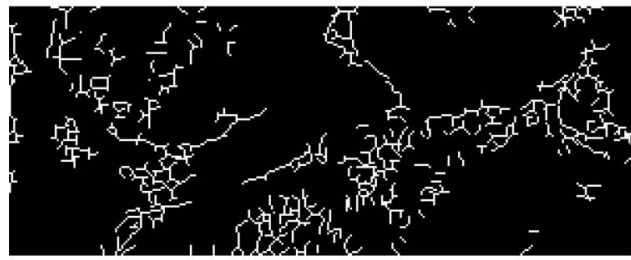

(d)

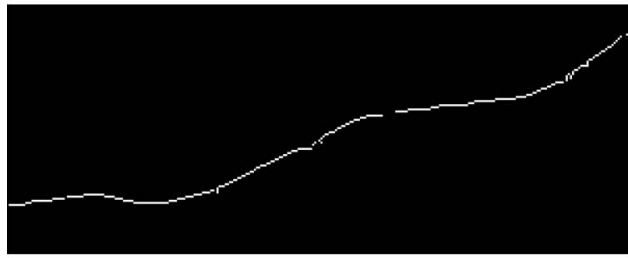

(f)

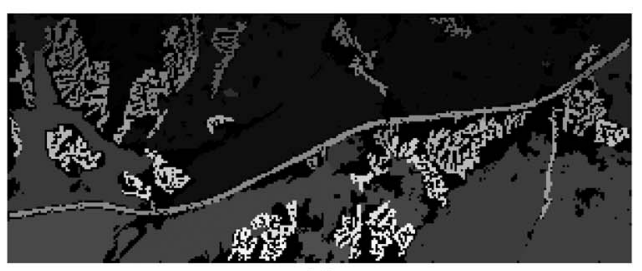

(c)

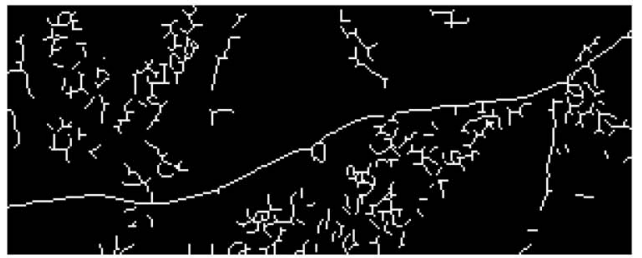

(e)

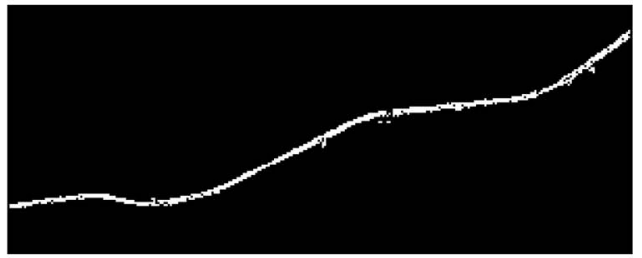

(g)

Fig. 8. Road extraction from a satellite image. (a) Satellite image containing a road with $300 \times 120$ pixels. (b) LEGION segmentation result with bright road thresholding. (c) LEGION segmentation result with dark road thresholding. (d) Candidate medial axis points in (b). (e) Candidate medial axis points in (c). (f) Grouped medial axis points based on (e). (g) Road extraction result. The parameters used here are $W_{z}=60, \theta_{p}=45, N_{p}=50$, and $W_{z g}=0.85$.

The first test image is shown in Fig. 8(a), which is an Earth Observing-1 (EO-1) satellite image with a resolution of 30 m collected at Silver Lake in Mojave Desert, California. This image contains one main road. Two segmentation results using two kinds of thresholding are shown in Fig. 8(b) and (c), where each gray value indicates a distinct segment. The candidate medial axis points are then selected based on both segmentation results, shown in Fig. 8(d) and (e). The result of grouping the candidate medial axis points extracted from Fig. 8(e) is shown in Fig. 8(f). Because of the absence of leaders in the road grouping stage, no road medial axis points are extracted based on Fig. 8(d). The restored road region is presented in Fig. 8(g). The road is successfully extracted. Worth mentioning is that, in the image, a short road with the corresponding segment obtained in Fig. 8(c) is not extracted in the final result, which is due to the parameter settings in leader selection. This road could be easily extracted by loosening parameter constraints so that some leaders of this road are stimulated.

The next test image is an IKONOS satellite image with a resolution of $4 \mathrm{~m}$ collected at Tampa, Florida. This image contains two major roads, as shown in Fig. 9(a). Two segmentation results are shown in Fig. 9(b) and (c), and Fig. 9(d) and (e) shows the corresponding road extraction results. In Fig. 9(d), two roads are produced separately, indicated by different gray values. As can be seen, both roads are clearly extracted. Since the separators of the right road appear as thin, elongated, and homogeneous regions, coinciding with the assumption of roads, these regions are extracted as dark roads, as shown in Fig. 9(e). Eliminating regions of this kind entails detecting more complex characteristics of road properties. These as well as other results not shown demonstrate that the proposed approach for automatic road extraction is successful in extracting roads from satellite imagery.

The experiments with different parameter settings show that qualitatively similar results can be obtained when parameter values are chosen within certain ranges. As already mentioned, there are four parameters that need to be tuned in the method. The parameters $\theta_{p}$ and $N_{p}$ are used for generating leaders in the road grouping stage, where $\theta_{p}$ is chosen according to $N_{p}$. $W_{z}$ and $W_{z g}$ are two inhibition weights of the LEGION models in the first and third stages. The parameter value ranges for the images in Figs. 8(a) and 9(a) are summarized in Table I.

\section{Comparisons}

Mayer et al. [15] set up a European Spatial Data Research (EuroSDR) test to compare different approaches for automatic road extraction. To put the performance of our algorithm in 


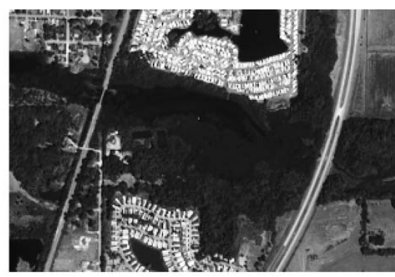

(a)

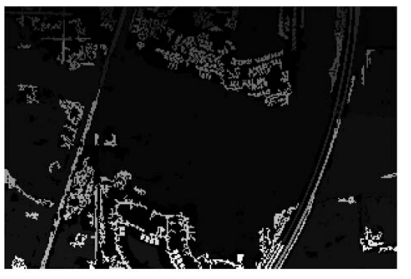

(b)

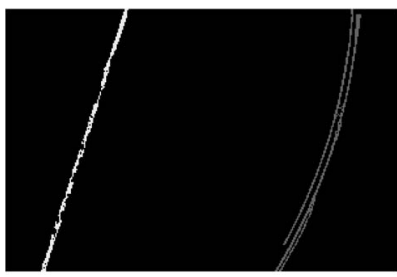

(d)

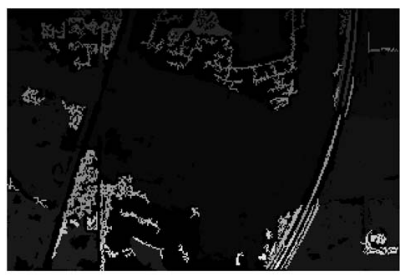

(c)

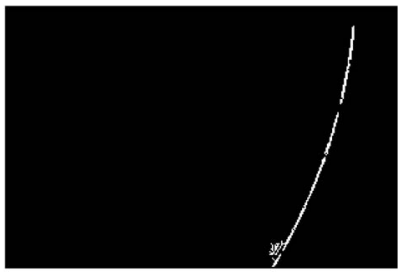

(e)
Fig. 9. Road extraction from another satellite image. (a) Satellite image containing two main roads with $300 \times 200$ pixels. (b) LEGION segmentation result with bright road thresholding. (c) LEGION segmentation result with dark road thresholding. (d) Road extraction result based on (b). (e) Road extraction result based on (c). The parameters used here are $W_{z}=40, \theta_{p}=55, N_{p}=$ 60 , and $W_{z g}=0.85$

TABLE I

VALID PARAMETER VALUE RANGES FOR IMAGES IN FIGS. 8(a) AND 9(a)

\begin{tabular}{ccc}
\hline \hline Parameter & Fig. 8(a) & Fig. 9(a) \\
\hline$N_{p}$ & $45-70$ & $45-70$ \\
$W_{z}$ & $60-100$ & $30-85$ \\
$W_{z g}$ & $0.8-0.95$ & $0.76-0.9$ \\
\hline \hline
\end{tabular}

perspective, the system was applied to the benchmark images used in the test, and the results were compared with those provided [15]. The experiments were performed using three $1600 \times 1600$ IKONOS images, the Ikonos3-Sub1, Ikonos3Sub2, and Ikonos1-Sub1, at the resolution of $2 \mathrm{~m}$. Each of the images has four spectral bands: red, green, blue, and infrared. In the experiments, the red band is utilized, which gives slightly better results than the other bands. Like other test participants, a Normalized Difference Vegetation Index was incorporated into the approach, which is first computed from the red and the infrared bands. After segmentation, if $80 \%$ of the pixels in a segment have positive values, the segment is considered to be a vegetation area and thus discarded in subsequent processing.

For automatic road extraction, two indices, completeness and correctness, are widely used to quantitatively evaluate extraction results, which were introduced by Wiedemann et al. [25]. Completeness is the percentage of the reference road pixels that lie within a buffer area around the extracted roads, and correctness is the percentage of the extracted road pixels that lie
TABLE II

RESUlT OF THE QUANTITATIVE EVALUATION

\begin{tabular}{|c|c|c|}
\hline Author & Completeness & Correctness \\
\hline & \multicolumn{2}{|l|}{ Ikonos3_Sub1 } \\
\hline Bacher & 0.81 & 0.87 \\
\hline Gerke_W & 0.8 & 0.65 \\
\hline Gerke_WB & 0.68 & 0.75 \\
\hline Hedman & 0.77 & 0.78 \\
\hline Malpica & 0.6 & 0.79 \\
\hline Proposed & 0.83 & 0.71 \\
\hline \multicolumn{3}{|c|}{ Ikonos3_Sub2 } \\
\hline Bacher & 0.86 & 0.89 \\
\hline Gerke_W & 0.75 & 0.52 \\
\hline Gerke_WB & 0.71 & 0.84 \\
\hline Hedman & 0.85 & 0.91 \\
\hline Malpica & 0.6 & 0.89 \\
\hline Proposed & 0.89 & 0.62 \\
\hline \multicolumn{3}{|c|}{ Ikonos1_Sub1 } \\
\hline Bacher & 0.34 & 0.66 \\
\hline Beumier & 0.48 & 0.69 \\
\hline Gerke_W & 0.27 & 0.41 \\
\hline Gerke_WB & 0.19 & 0.49 \\
\hline Hedman & 0.31 & 0.51 \\
\hline Malpica & 0.25 & 0.74 \\
\hline Zhang & 0.56 & 0.41 \\
\hline Proposed & 0.59 & 0.65 \\
\hline
\end{tabular}

within a buffer area around the reference roads. In the test, the buffer width is set to 5 pixels, and the corresponding reference data are provided in the form of the centerlines of the roads. Evaluation results of the test participants as reported in [15], as well as results from the proposed system, are shown in Table II. For each image, the best result in terms of the average of completeness and correctness scores is marked in bold.

Ikonos3-Sub1 and Ikonos3-Sub2 are two rural hilly scenes. Fig. 10(a) and (c) shows the red bands of the images, where the reference road data are marked in black. The extracted medial axis points from the proposed method are overlaid on the original images and displayed in Fig. 10(b) and (d). It can be seen from Table II that the results are the most complete for these two images. Although the correctness rates are lower than the best results, a close comparison indicates that a considerable portion of the extracted results, while not matched with the reference data, are very likely roads based on the image appearance.

Ikonos1-Sub1 is from an urban/suburban area in a hilly terrain. Fig. 11(a) shows the red band of the image with the reference road data. It has been stated [15] that this scene is very challenging for the existing approaches. A large number of roads are partially occluded or confused with surrounding objects. The extraction result from the proposed system is shown in Fig. 11(b), where many highly fragmented roads 


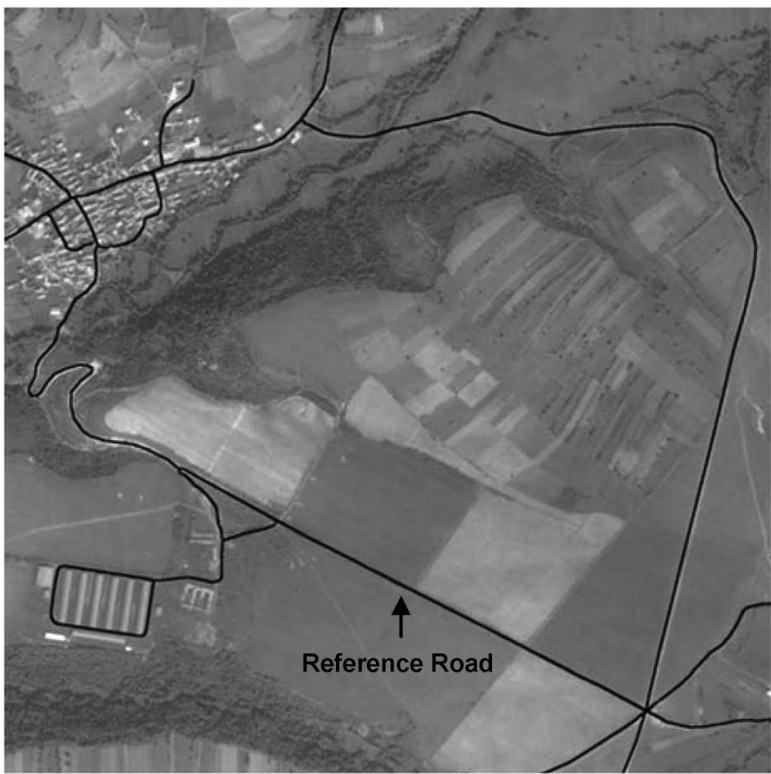

(a)

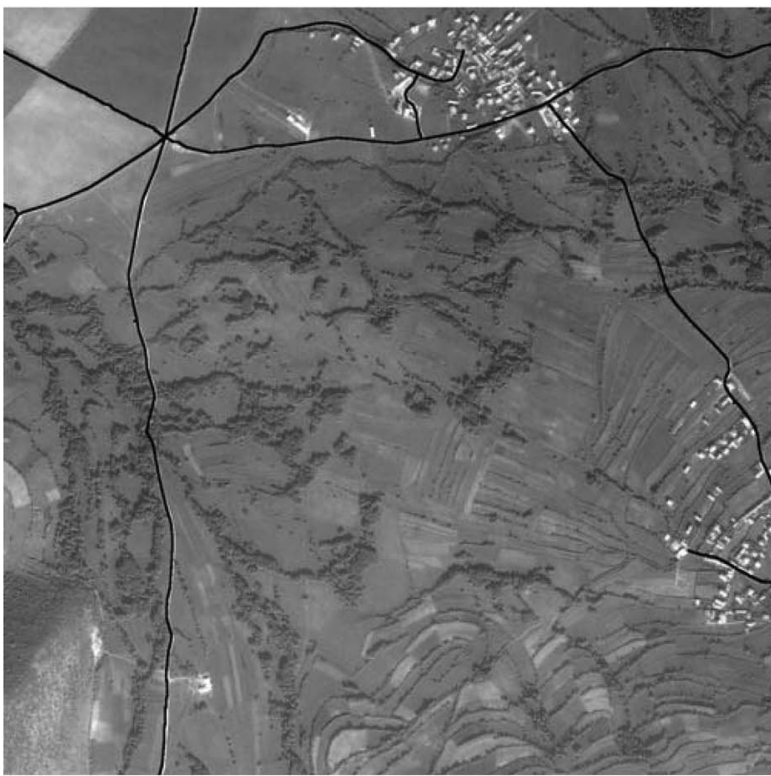

(c)

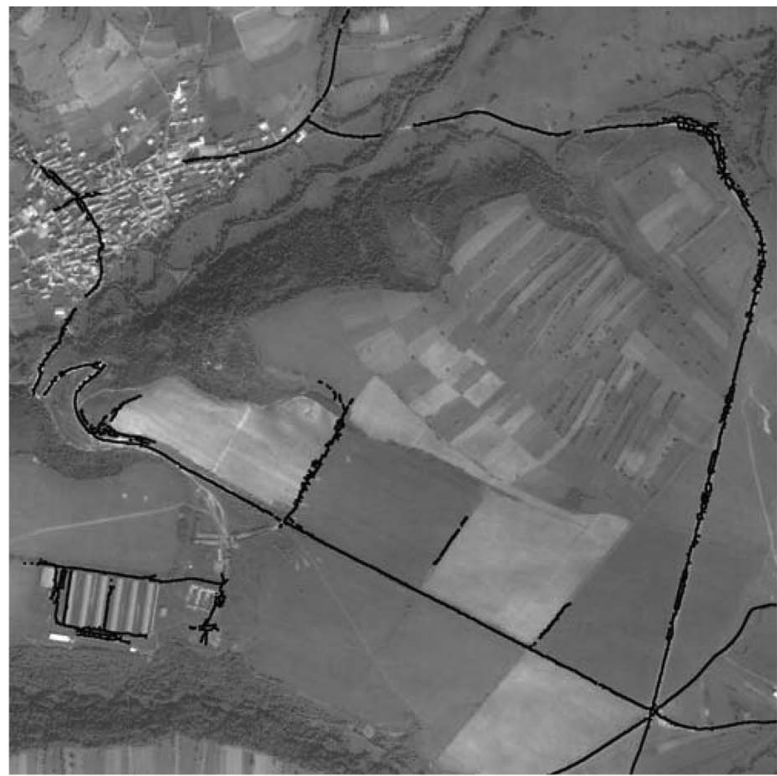

(b)

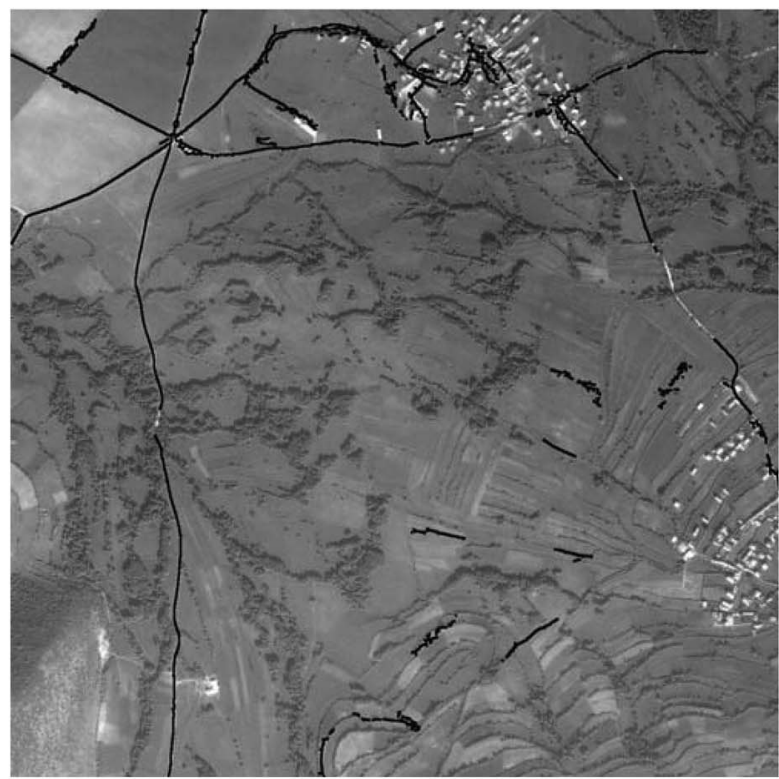

(d)

Fig. 10. Road extraction from Ikonos3-Sub1 and Ikonos3-Sub2. (a) Red band image of Ikonos3-Sub1 with reference data marked in black. (b) Extraction result from (a) using the proposed method with the parameters $W_{z}=90, \theta_{p}=50, N_{p}=40$, and $W_{z g}=0.85$. The extracted road centerlines, shown in black, are overlaid on the original image. (c) Red band image of Ikonos3-Sub2. (d) Extraction result from (c) using the proposed method with the parameters $W_{z}=90$, $\theta_{p}=50, N_{p}=40$, and $W_{z g}=0.75$.

are successfully extracted owing to the grouping stage. The quantitative measure in Table II shows that the proposed method yields the most complete result while the correctness rate is not far from the best.

This analysis as well as earlier ones [27] indicates that the proposed method produces very competitive road extraction results. The performance may be attributed to the use of the LEGION network with long-range horizontal connections, which can effectively extract smoothly aligned patterns.

\section{CONCLUDING REMARKS}

In this paper, a new automatic road extraction method based on LEGION has been presented. Three stages are employed for road extraction: image segmentation, selection of candidate medial axis points, and road grouping. While some aspects of the first two stages have been developed in previous studies, a number of improvements have been introduced in these stages. These improvements, together with the newly introduced road grouping stage, result in a novel automatic road extraction method. Quantitative evaluations confirm the effectiveness of this method.

The fundamental idea of the proposed method is to group similar features and segregate dissimilar ones. In the first stage, the gray level is used as a feature, which can be interpreted as similarity, and in the third stage, orientation is taken as a feature, which can be interpreted as good continuation. Both features are encoded in the LEGION model, which uses 


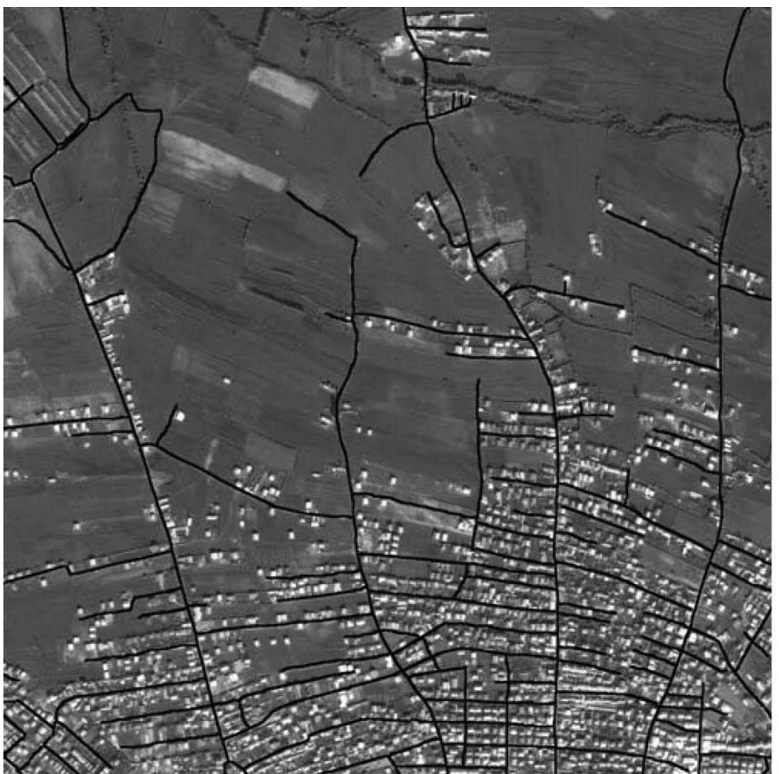

(a)

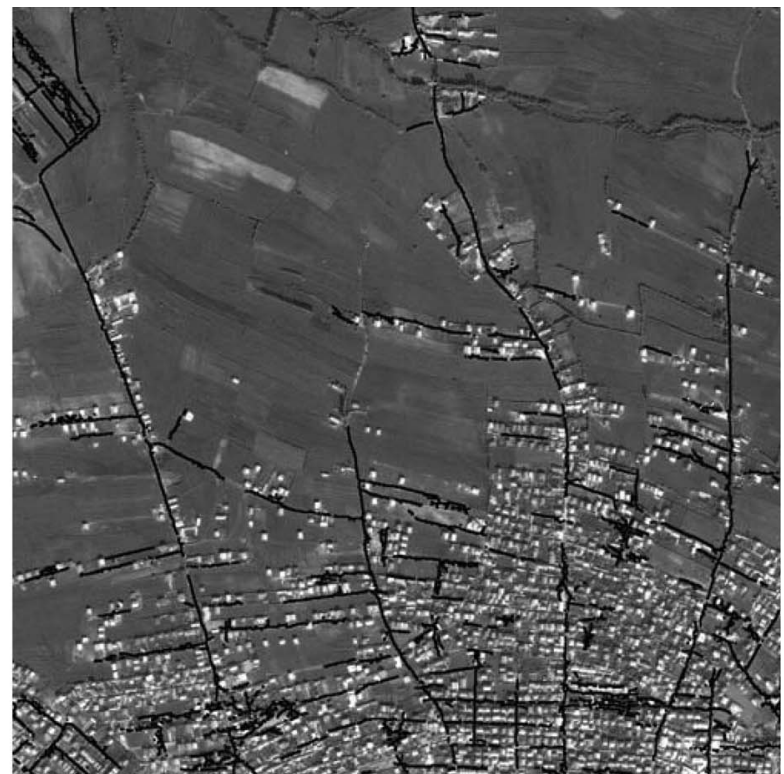

(b)

Fig. 11. Road extraction from Ikonos1-Sub1. (a) Red band image of Ikonos1-Sub1 with reference road data marked. (b) Extraction result using the proposed method with the parameters $W_{z}=60, \theta_{p}=35, N_{p}=40$, and $W_{z g}=0.85$.

dynamics of oscillatory correlation to achieve grouping. The generality of the underlying idea implies that the method could be used in a wide range of applications where region and boundary information need to be considered.

While the proposed method is not very sensitive to particular choices of parameters, the experiments show that fine tuning of parameters could further improve extraction results. Future work needs to address the issue of automatically determining the optimal parameters for different types of images (see, e.g., [7]).

\section{REFERENCES}

[1] J. Amini, M. R. Saradjian, J. A. R. Blais, C. Lucas, and A. Azizi, "Automatic road-side extraction from large scale imagemaps," Int. J. Appl. Earth Observation Geoinform., vol. 4, no. 2, pp. 95-107, Nov. 2002.

[2] M. Amo, F. Martinez, and M. Torre, "Road extraction from aerial images using a region competition algorithm," IEEE Trans. Image Process., vol. 15, no. 5, pp. 1192-1201, May 2006.

[3] D. Attali, J.-D. Boissonnat, and H. Edelsbrunner, "Stability and computation of the medial axis-A state-of-the-art report," in Mathematical Foundations of Scientific Visualization, Computer Graphics, and Massive Data Exploration, T. Möller, B. Hamann, and B. Russell, Eds. New York: Springer-Verlag, 2007.

[4] U. Bacher and H. Mayer, "Automatic road extraction from multispectral high resolution satellite images," in Proc. Int. Archives Photogramm. Remote Sens. Spatial Inf. Sci., 2005, vol. 36, pp. 29-34, 3/w24.

[5] M. Barzohar and D. B. Cooper, "Automatic finding of main roads in aerial images by using geometric stochastic models and estimation," IEEE Trans. Pattern Anal. Mach. Intell., vol. 18, no. 7, pp. 707-721, Jul. 1996.

[6] A. Baumgartner, C. Steger, H. Mayer, W. Eckstein, and H. Ebner, "Automatic road extraction based on multi-scale, grouping, and context," Photogramm. Eng. Remote Sens., vol. 65, no. 7, pp. 777-785, 1999.

[7] K. Chen, "Adaptive smoothing via contextual and local discontinuities," IEEE Trans. Pattern Anal. Mach. Intell., vol. 27, no. 10, pp. 1552-1567, Oct. 2005.

[8] K. Chen and D. L. Wang, "A dynamically coupled neural oscillator network for image segmentation," Neural Netw., vol. 15, no. 3, pp. 423-439, Apr. 2002

[9] K. Chen, D. L. Wang, and X. Liu, "Weight adaptation and oscillatory correlation for image segmentation," IEEE Trans. Neural Netw., vol. 11, no. 5, pp. 1106-1123, Sep. 2000.
[10] T. K. Dey and W. Zhao, "Approximating the medial axis from the Voronoi diagram with a convergence guarantee," Algorithmica, vol. 38, no. 1 , pp. 179-200, Oct. 2003.

[11] H. Mayer, S. Hinz, and U. Stilla, "Automated extraction of roads, buildings and vegetation from multi source data," in Advances in Photogrammetry, Remote Sensing and Spatial Information Sciences: 2008 ISPRS Congress Book, Z. Li, J. Chen, and E. Baltsavias, Eds. New York: Taylor \& Francis, 2008.

[12] X. Liu, K. Chen, and D. L. Wang, "Extraction of hydrographic regions from remote sensing images using an oscillator network with weight adaptation," IEEE Trans. Geosci. Remote Sens., vol. 39, no. 1, pp. 207211, Jan. 2001

[13] D. J. Field, A. Hayes, and R. F. Hess, "Contour integration by the human visual system: Evidence for a local 'Association Field'," Vision Res., vol. 33, no. 2, pp. 173-193, Jan. 1993.

[14] C. Gros, Complex and Adaptive Dynamical Systems: A Primer. Berlin, Germany: Springer-Verlag, 2008.

[15] H. Mayer, S. Hinz, U. Bacher, and E. Baltsavias, "A test of automatic road extraction approaches," Int. Archives Photogramm., Remote Sens., Spatial Inf. Sci., vol. 36, no. 3, pp. 209-214, 2006.

[16] X. Niu, "A semi-automatic framework for highway extraction and vehicle detection based on a geometric deformable model," ISPRS J. Photogramm. Remote Sens., vol. 61, no. 3/4, pp. 170-186, Dec. 2006.

[17] R. L. Ogniewicz and O. Kübler, "Hierarchic Voronoi skeletons," Pattern Recognition, vol. 28, no. 3, pp. 343-359, Mar. 1995.

[18] P. Parent and S. W. Zucker, "Trace inference, curvature consistency, and curve detection," IEEE Trans. Pattern Anal. Mach. Intell., vol. 11, no. 8, pp. 823-839, Aug. 1989.

[19] C. Poullis and S. You, "Remote delineation and geometric modeling of road networks," ISPRS Journal of Photogramm. Remote Sens., vol. 65, no. 2, pp. 165-181, Mar. 2010

[20] I. Rock and S. Palmer, "The legacy of Gestalt psychology," Sci. Amer., vol. 263, no. 10, pp. 84-90, 1990.

[21] D. Terman and D. L. Wang, "Global competition and local cooperation in a network of neural oscillators," Phys. D, vol. 81, no. 1/2, pp. 148-176, Feb. 1995.

[22] D. L. Wang, "Object selection based on oscillatory correlation," Neural Netw., vol. 12, no. 4/5, pp. 579-592, Jun. 1999.

[23] D. L. Wang, "The time dimension for scene analysis," IEEE Trans. Neural Netw., vol. 16, no. 6, pp. 1401-1426, Nov. 2005.

[24] D. L. Wang and D. Terman, "Image segmentation based on oscillatory correlation," Neural Comput., vol. 9, no. 4, pp. 805-836, May 1997.

[25] C. Wiedemann, C. Heipke, and H. Mayer, "Empirical evaluation of automatically extracted road axes," in Proc. CVPR Workshop Empirical Evaluation Methods Comput. Vis., 1998, pp. 172-187. 
[26] S.-C. Yen and L. H. Finkel, "Extraction of perceptually salient contours by striate cortical networks," Vision Res., vol. 38, no. 5, pp. 719-741, Mar. 1998.

[27] J. Yuan, D. L. Wang, B. Wu, L. Yan, and R. Li, "Automatic road extraction from satellite imagery using LEGION networks," in Proc. Int. Joint Conf. Neural Netw., 2009, pp. 3471-3476.

[28] J. Zhou, W. F. Bischof, and T. Caelli, "Robust and efficient road tracking in aerial images," in Proc. Int. Archives Photogramm., Remote Sens., Spatial Inf. Sci., 2005, vol. 36, pp. 35-40, 3/w24.

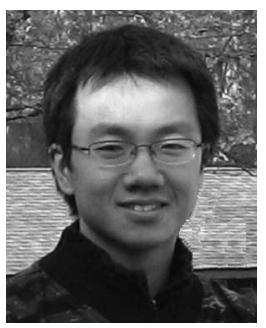

Jiangye Yuan received the M.S. degree in computer science and engineering from The Ohio State University, Columbus, in 2009, where he is currently working toward the Ph.D. degree in geodetic science.

His research interests include image segmentation, neural networks, and pattern recognition with applications in remote sensing and geospatial analysis.

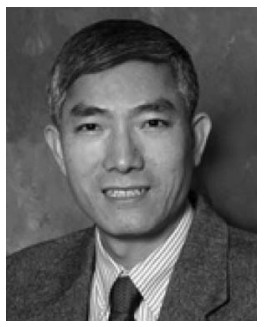

DeLiang Wang (M'90-SM'01-F'04) received the B.S. and M.S. degrees in computer science from Peking (Beijing) University, Beijing, China, in 1983 and 1986, respectively, and the Ph.D. degree in computer science from the University of Southern California, Los Angeles, in 1991.

From July 1986 to December 1987, he was with the Institute of Computing Technology, Academia Sinica, Beijing. Since 1991, he has been with the Department of Computer Science and Engineering and the Center for Cognitive Science, The Ohio State University, Columbus, where he is currently a Professor. From October 1998 to September 1999, he was a Visiting Scholar in the Department of Psychology, Harvard University, Cambridge, MA. From October 2006 to June 2007, he was a Visiting Scholar at Oticon A/S, Denmark. His research interests include machine perception and neurodynamics. He is a Co-Editor-in-Chief of Neural Networks.

Dr. Wang was the recipient of the Office of Naval Research Young Investigator Award in 1996, the 2005 Outstanding Paper Award from the IEEE Transactions on Neural Networks, and the 2008 Helmholtz Award from the International Neural Network Society.

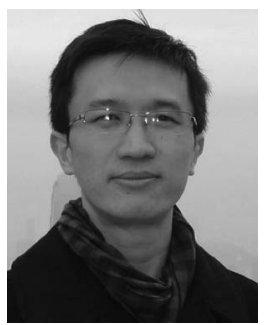

Bo $\mathbf{W u}$ received the $\mathrm{Ph} . \mathrm{D}$. degree in photogrammetry and remote sensing from Wuhan University, Wuhan, China, in 2006.

From July 2006 to July 2009, he was a Postdoctoral Researcher and a Research AssociateEngineering with the Mapping and GIS Laboratory, The Ohio State University, Columbus. From July 2009 to present, he is an Assistant Professor with the Department of Land Surveying and Geo-Informatics, The Hong Kong Polytechnic University, Kowloon, Hong Kong. His research interests include automated photogrammetry, planetary mapping, machine vision, and 3-D Geographic Information System (GIS).

Dr. Wu was a recipient of the Duane C. Brown Senior Award (Photogrammetry) in 2009.

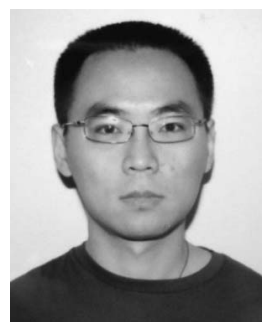

Lin Yan received the B.S. degree in surveying engineering and the M.S. degree in photogrammetry and remote sensing from Tongji University, Shanghai, China, in 2002 and 2005, respectively. He is currently working toward the Ph.D. degree in the Department of Civil and Environmental Engineering and Geodetic Science, The Ohio State University, Columbus.

His research interests include high-precision image matching, image segmentation and classification of multispectral and hyperspectral remote sensing images, and nonlinear dimensionality reduction of hyperspectral image using manifold learning techniques.

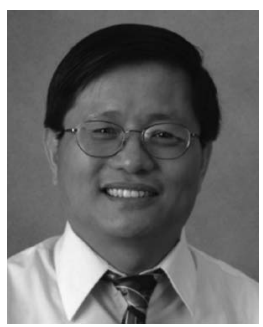

Rongxing (Ron) Li received the B.S. and M.S. degrees in surveying engineering from Tongji University, Shanghai, China, in 1982 and 1984, respectively, and the $\mathrm{Ph} . \mathrm{D}$. in photogrammetry and remote sensing from the Technical University of Berlin, Berlin, Germany, in 1990.

He was an Assistant and Associate Professor with the Department of Geomatics Engineering, The University of Calgary, Calgary, AB, Canada, from 1994 to 1996. In 1996, he moved to The Ohio State University, becoming a Full Professor in 2001. His research interests include photogrammetric engineering and geomatics applications, planetary exploration, digital mapping, spatial data structures, and coastal and marine GIS. He is a Participating Scientist of the Mars Exploration Rover and the Lunar Reconnaissance Orbiter National Aeronautics and Space Administration (NASA) missions and a Member of the Mars Exploration Program Geodesy and Cartography Working Group. In addition, he is a Science Team Member of the Panoramic Camera (PanCam) system of the European Space Agency ExoMars mission. He is the Editor-in-Chief of the international journal Marine Geodesy.

Dr. Li is an American Society of Civil Engineering Fellow and has been the recipient of numerous academic and professional awards, including several NASA Group Achievement Awards, the Pacific Congress on Marine Science and Technologies Service Award, and best paper awards from the International Society for Photogrammetry and Remote Sensing, the American Society of Photogrammetry and Remote Sensing, and Chinese Professionals of Geographic Information System. In addition, he is a recipient of the Duane C. Brown Photogrammetry Award, the Lumley Research Award, and the Innovators Award presented by The Ohio State University. 